\title{
Spray Inoculation of Plates in the Detection of Antagonistic Micro-organisms
}

\author{
By A. WILSKA \\ The Wihuri Institute, Helsinki
}

SUMMARY: By means of a simple atomizer the surface of agar media may be sprayed with a suspension of micro-organisms, so that an even growth is obtained. The method is suitable for the seeding of plates for the assay of antimicrobial substances. It is particularly valuable for the detection of microbial antagonisms, for a test organism may be seeded on to established plate cultures of other organisms, without disturbing the bacterial colonies already present on the agar surface.

The discovery of penicillin has in recent years stimulated the search for other antibiotics. A commonly used method for discovering antagonistic microorganisms is to distribute a mixed culture from soil, manure, etc., on to the surface of a plate seeded with a suitable test organism. After incubation, a clear zone free from the test bacterium indicates the presence of an antagonist. The drawbacks of this technique are, first, that the potential antagonists, especially if they develop slowly, may be choked by the growth of the test organism, and secondly, a non-lytic antibiotic may leave no visible record of its action. The obvious remedy is to allow the organisms from the soil or other suspension to develop for some time before seeding with the test organism. It is rarely possible to seed a plate with a suspension of the test organism and drain off the excess fluid, as bacteria from the already developed colonies will be spread over the plate. On the other hand, to spread the suspension between the colonies by means of a loop or spreader without touching them is always tedious and frequently impracticable.

A satisfactory solution of the problem is to allow the potential antagonists to develop for a time, and then to spray a suspension of the test organism on to the plate. A device which we have used for this purpose is shown in Fig. 1. The conical flask $\boldsymbol{A}$ has a hole cut in the bottom slightly smaller than the Petri dish. The sprayer itself, the construction of which will be understood from the figure, is wrapped with cotton or cellulose tape and fitted snugly into the neck of the flask. Air is blown by means of a bulb through the cotton-wool filter $B$ in tube $C$, the lower part of which is drawn out to a jet. Tube $D$, the top of which is sealed to $C$, is drawn out to a fairly fine point so that surface tension will prevent the few drops of bacterial suspension which are placed in $D$ from running out by gravity; it must be accurately alined with $C$ if a nebulous spray free from coarse droplets is to be obtained.

To use the sprayer, a few drops of bacterial suspension are placed in tube $D$, the flask $A$ is placed on the Petri dish from which the cover has been removed, and the bulb is manipulated once or a few times. A beautifully even growth of bacteria is obtained if as little as one drop of suspension is sprayed in this way. 
With highly pathogenic bacteria it is advisable to wrap the joint between flask and Petri dish with cotton-wool; also, sufficient time should be allowed for the fine droplets to settle before removing the flask from the dish.

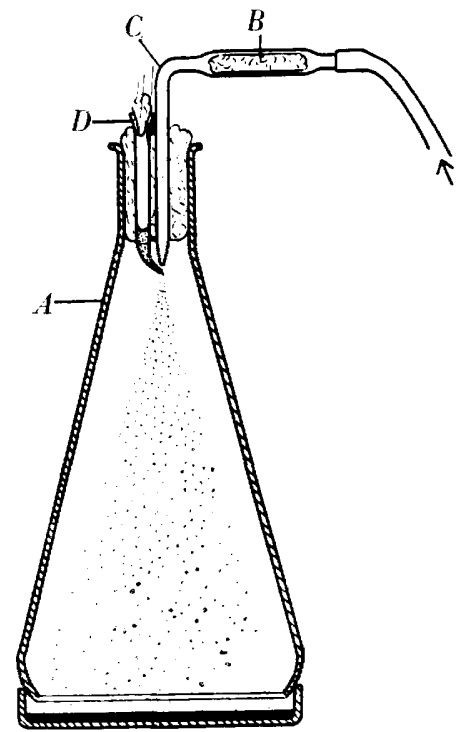

Fig. 1. Median vertical section through the spraying apparatus.

Plates seeded in this way require little or no drying. The technique has also been found convenient in the first stage in the search for antagonists, namely, for distributing the suspension of soil or other material under test. It is also suitable for surface seeding the agar for cylinder- or cup-plate assay.

(Received 3 March 1947) 